\title{
El concepto de explicación en las ciencias sociales
}

\author{
Lluís Ballester Brage \\ Antonio J. Colom Cañellas \\ Universidad de las Islas Baleares. Facultad de Educación \\ Edificio Guillem Cifre de Colonya \\ Carretera de Valldemossa, km. 7,5. 07071 Palma de Mallorca \\ lluis.ballester@uib.es \\ wvilanova@ono.com
}

\section{Resumen}

Este artículo pone de relieve la importancia que tiene para las ciencias sociales pensar la teoría de la explicación. Más allá de la tradición sociológica que ratifica sin reservas la legitimidad de la teoría clásica de la explicación o que acepta sin reservas el individualismo metodológico, este artículo asume una reflexión epistemológica dirigida a interrogarse sobre las consecuencias de aceptar una u otra concepción. Intentaremos en estas páginas una aproximación crítica a los últimos desarrollos que tal cuestión ha merecido, fundamentalmente, de los neomarxistas analíticos británicos.

Palabras clave: explicación, explicación nomológica, explicación estadística, explicación por procesos, explicación funcional, explicación teleológica, mecanismos.

\section{Abstract. The Explanation Concept in the Social Sciences}

This article emphasizes the importance which the social science assigns to think the theory of explanation. Beyond the sociological tradition that ratifies without reserves the legitimacy of the classic theory of the explanation or that accepts without reserves the methodological individualism, this article assumes a epistemological reflexion aiming to examine the consequences of accepting one or other conception. We will try in these pages an approximation to the last developments that such question has deserved, fundamentally, of the British analytic neomarxists.

Key words: explanation, explanation by norms, statistical explanation, explanation by processes, functional explanation, finalist explanation, mechanisms.

\section{Sumario}

1. Acerca de la explicación en las ciencias sociales

2. El individualismo metodológico de Jon Elster y la crítica de los modelos explicativos holísticos
4. La explicación funcional reformada en el materialismo histórico

5. Crítica al individualismo metodológico

6. Bibliografía

3. La explicación por mecanismos 


\section{Acerca de la explicación en las ciencias sociales}

Definir lo que es una explicación no conlleva mayor dificultad, puede decirse que significa hablar de una cosa para darla a conocer a otros a fin de que la comprendan. Ahora bien, cuando llevamos la explicación al campo de la ciencia el problema se hace más complejo, por ejemplo, una explicación en física incluye, al menos, dos elementos fundamentales; en primer lugar, unas leyes que forman parte de una teoría más amplia y, en segundo lugar, un lenguaje matemático en el que estas leyes se expresan. Las ciencias de la vida y las ciencias sociales tienen otra manera de considerar la explicación. En la historia de la ciencia se puede observar cómo las diversas tradiciones han desarrollado formas de explicación diferenciadas. Intentaremos a continuación mostrar algunas de las tradiciones «explicativas» principales. ${ }^{1}$

\section{A. La explicación en ciencias de la materia (el caso de la física)}

Uno de los autores que con más claridad ha planteado la problemática de la explicación ha sido Bunge (1983), quien ha clarificado su sentido al afirmar que «La ciencia [...] intenta explicar los hechos en términos de leyes, y las leyes en términos de principios. Los científicos no se conforman con descripciones detalladas; además de inquirir cómo son las cosas, procuran responder a por qué: por qué ocurren los hechos, cómo ocurren y no de otra manera. La ciencia deduce proposiciones relativas a hechos singulares a partir de leyes generales, y deduce las leyes a partir de enunciados nomológicos aún más generales (principios)» (Bunge 1983: 30). Un concepto fundamental de la explicación en las ciencias de la naturaleza es, por tanto, la noción de ley. Bunge ha asignado diferentes significados al termino ley, pero aquí puede interpretarse como toda relación constante y objetiva en la naturaleza, en la mente o en la sociedad (Bunge 1973: 69 y s.). Es importante señalar, además, que esas leyes resultan significativas en el contexto más general de una teoría.

1. En este trabajo se consideran tres tipos de ámbitos científicos cuyos niveles de seguridad teórica son cada vez menos fiables debido en parte a la función de cambio a que están sometidos sus objetos de conocimiento:

- Ciencias de la materia (física, geología, química, etc.): su objeto no cambia a lo largo del tiempo, tienen plena capacidad para experimentar con sus objetos de conocimiento y desarrollan tecnologías que permiten el control de la naturaleza.

- Ciencias de la vida (biología, ciencias médicas, etc.): su objeto cambia en largos ciclos temporales, ya que todos los seres vivos están sometidos a la evolución; tienen una cierta limitación en su capacidad para experimentar, no pudiendo generar daños sobre los seres humanos; sus tecnologías permiten el control sobre la naturaleza viva.

- Ciencias sociales y humanas (psicología, pedagogía, sociología, economía, historia, antropología, etc.): su objeto cambia en ciclos temporales cortos, ya que tratan de sujetos y sociedades humanas; tienen mayores dificultades para hacer experimentación, y en algunas disciplinas ni siquiera hay posibilidad (por ejemplo: historia), además hay distorsiones producidas por la intencionalidad humana y/o por la subjetividad. Sus tecnologías son de eficacia limitada. 
Concretando más, puede decirse que una explicación física consiste en demostrar que un fenómeno dado es una consecuencia de leyes ya aceptadas. La fuerza de la explicación reposa en dos puntos esenciales:

i) La necesidad inherente a todo esquema explicativo, en la medida en que se trata de una explicación lógica.

ii) La aceptabilidad de la teoría total de la que forman parte las leyes utilizadas en el esquema explicativo. (García 1973: 114).

En la explicación física se pueden encontrar, por tanto, tres aspectos fundamentales, a saber: el fenómeno que se trata de explicar, la ley en función de la cual se explica y la teoría en la que se insertan esas leyes. Entre otras consideraciones, lo que puede aprovecharse del modelo de las explicaciones físicas es que los fenómenos aparecen insertos en marcos más amplios que les permiten entrar en relación con otros fenómenos, es decir, se comprenden unos en relación con otros.

\section{B. La explicación en ciencias de la vida (el caso de la biología)}

La explicación en una ciencia no es totalmente ajena a la concepción que tengamos de su objeto ya que éste depende de la perspectiva desde la que los científicos «entiendan» la realidad. Resulta muy significativo que ésta sea la opinión de un biólogo:

Contrariamente a lo que se cree a menudo, el camino de la ciencia no consiste sólo en observar, en acumular datos experimentales para deducir una teoría. Es perfectamente posible observar un objeto durante años sin extraer jamás la menor observación de interés científico [...] Si la ciencia evoluciona es porque un aspecto todavía desconocido de las cosas se desvela de repente; no siempre como consecuencia de la aparición de instrumentos nuevos, sino gracias a un nuevo modo de examinar los objetos, de considerarlos bajo un ángulo nuevo. Esa mirada está necesariamente guiada por una cierta idea de lo que pueda ser «la realidad». Implica siempre una cierta concepción de lo desconocido, de esa zona situada más allá de lo que la lógica y la experiencia autorizan a creer (Jacob 1981: 29-30).

F. Jacob, premio Nobel de medicina en 1965, incide en la cuestión al señalar que es en las ciencias de la vida en donde esas nociones previas condicionan más los resultados. Nociones que en la biología contemporánea parecen obedecer a dos tendencias: por una parte, quienes hacen emerger la vida de un sustrato material y, por otra, quienes reducen lo vital a lo físico-químico (Cellerier 1980), lo que supone plantear dos perspectivas diferentes de una misma realidad.

Tratando de superar esa dualidad, Cellerier, que fue colaborador directo de Piaget, se refiere a los organismos unicelulares como una especie de fábricas itinerantes que buscan sus propias materias primas, capaces de autorregularse y asegurarse, así, su propio mantenimiento. Se trata de sistemas que desarrollan 
interacción con su medio, pues en caso contrario no sería posible la conservación del sistema. En este proceso se advierte, según Cellerier, una doble cara del organismo. Por una parte, nos encontramos ante un sistema sometido a las mismas leyes que su medio, que es a su vez un sistema más amplio que el del propio organismo; por la otra, cuando se produce esa interacción, existe un intercambio con el medio y se produce una respuesta. Teniendo en cuenta ambos fenómenos, Cellerier concluye que en biología se mezclan dos tipos de explicaciones: la causal y la funcional.

Se trataría de una complementariedad de formas de explicación. La explicación causal supondría situarse en el nivel material de los procesos físico-químicos, sin hacer intervenir los fines. Por el contrario, en una explicación funcional se prescindiría del sustrato material subyacente para preocuparse de la organización de los medios y los fines. Una explicación funcional se centra en un conjunto de elementos individuales con funciones propias que actúan conjuntamente para engendrar una función global determinada; es decir, en la explicación causal los acontecimientos se explicarían por sus antecedentes, mientras que en la funcional se explican por el papel que cumplen en un sistema.

Como afirma Pinillos (1983), reconocer realidades organizadas en niveles que difieren no sólo en complejidad sino en la cualidad configurativa, obliga a reconocer diversas formas de explicación, por lo que las ciencias sociales podrían reconocer la necesidad de desarrollar explicaciones alternativas a las causales, asumiendo una diversidad explicativa de acuerdo con su objeto de conocimiento.

\section{La explicación en ciencias sociales}

Ante los distintos modelos de explicación, ¿cuál es el camino que pueden tomar las ciencias sociales?; ¿¿deberá aproximarse a la ciencia física o, por el contrario, se pueden dar modelos que aceptan la especificidad del sujeto, de los procesos, de las relaciones, de las organizaciones, frente al organismo vivo y de éste frente a la realidad física?

Está claro que las teorías o las hipótesis, aunque se refieran correctamente a los datos, no producen siempre explicaciones causales en el sentido en que las producen las ciencias de la materia y de la vida, siendo éste un déficit epistemológico, pues, como afirmara Rappoport (1995), los investigadores en ciencias sociales a menudo aportan explicaciones racionales, rigurosas y coherentes de cómo ocurre un hecho sin conseguir explicar por qué ocurre. Se sigue pues con el tópico teórico, basado en una lectura superficial de Von Wright (1979), o tal vez de la herencia no asumida del historicismo de Dilthey, de que la dicotomía entre comprensión y explicación sólo tiene solución mediante el triunfo de la explicación causal. Pero este supuesto es parcial, no presta atención a la diversidad de posiciones teóricas sobre la explicación, al fracaso histórico del neopositivismo, ni a las modalidades de explicación que se observan en los artículos científicos. Para poder abrir las perspectivas del 
debate que plantearemos sobre este tema, se presentarán algunas de las principales modalidades de explicación y algunas de las posiciones sobre la explicación en ciencias sociales.

Prestando atención a algunos de los posicionamientos explicativos más conocidos en ciencias sociales, se puede afirmar sin duda que hay dos grandes opciones: la opción básica es, por un lado, la que entiende la explicación como causalidad a la manera de las ciencias naturales; ${ }^{2}$ por otro lado, la que rechaza los modelos deterministas en las ciencias sociales, aceptando las explicaciones estadísticas y las explicaciones estructural-funcionales, y que por tanto, consideran que las explicaciones son diferentes en las ciencias sociales. Las dos concepciones remiten a argumentaciones metodológicas distintas, obviamente, sus posiciones epistemológicas influyen en sus opciones metodológicas. A título de ejemplo, prestaremos atención a dos autores, Piaget y Skinner, que han tratado de explicar la conducta con planteamientos diferentes: Skinner desde una modalidad del individualismo metodológico y Piaget desde una perspectiva holista.

Skinner (1970: 40) no ha dudado en aplicar a las ciencias de la conducta el mismo tipo de explicación que a cualquier otra ciencia de la naturaleza. Para Skinner las características básicas de la ciencia no están limitadas a ninguna disciplina especial. Cuando estudiamos física, química o biología, estudiamos acumulaciones organizadas de información sobre hechos. Skinner atribuye a la ciencia, en términos muy generales, dos tipos de características: (i) por una parte, la ciencia supone un conjunto de actitudes, es decir, una determinada disposición para tratar con los hechos; y (ii) por otra parte, realiza un determinado tratamiento de esos hechos. La explicación consiste en la búsqueda de un orden de uniformidades, de relaciones válidas entre los hechos. Por tanto, las leyes que se refieren a la conducta no tienen por qué ser diferentes de las que explican cualquier hecho de la naturaleza. La conducta, y el mundo físico se sitúan en un mismo plano. A partir de aquí pretenderá estudiar las causas de la conducta humana:

Una "causa» equivale a un cambio en una variable independiente y un efecto, a un cambio en una variable dependiente. La antigua relación causa-efecto se convierte en una relación funcional; estos nuevos términos no indican cómo la causa produce el efecto, se limitan simplemente a afirmar que hechos diferentes tienden a producirse en un cierto orden (Skinner 1970: 50).

Conviene resaltar dos puntos. En primer lugar, que establece relaciones entre causas y efectos. Como señala Pinillos refiriéndose a la explicación skinneriana, las causas de la conducta son externas, no orgánicas y menos mentales; es decir, lo que se considera causa son los antecedentes de la conducta. En

2. Sólo se aceptan modelos de explicación determinista, es decir, la determinación por causas necesarias y suficientes, aunque también se reconoce el papel de las explicaciones basadas en modelos estadísticos (probabilísticos). 
segundo lugar, el texto se refiere a un «análisis funcional». Esa función tiene muy poco que ver con la noción de función o con la explicación funcional a la que antes se ha hecho referencia, ya que se refiere a la noción matemática de función (Pinillos 1983); $y=f(x)$, es decir, el cambio en una variable va seguido de una variación en la otra. En resumen, lo que interesa resaltar aquí es que para Skinner la explicación en ciencias del comportamiento no tiene por qué ser diferente de la que se lleva a cabo en las ciencias de la naturaleza.

Por su parte, Piaget se sitúa en un contexto completamente diferente al reconocer la especificidad de las ciencias del comportamiento. Para Piaget, el tipo de explicación que se adopte depende, en último extremo, de una determinada concepción del objeto de conocimiento, es decir, el problema metodológico de la explicación debe ser también interpretado en términos epistemológicos. Piaget acepta una amplia variedad de formas de explicación, según la diversidad de objetos de conocimiento, desde explicaciones causales hasta explicaciones evolutivas o procesuales, pasando por las explicaciones funcionales, siempre teniendo como horizonte la construcción del conocimiento objetivo.

Las posiciones de Skinner y Piaget pueden servir de ejemplo de la diversidad de posiciones enfrentadas. En el contexto de las ciencias sociales no están resueltos los problemas relativos a la explicación, se dispone de una variada tipología de opciones para desarrollar explicaciones. Las posiciones reduccionistas sólo aceptarán como válidas las dos primeras formas de explicación (nomológico-deductiva y estadística), tomando como control de la validez de las explicaciones la posibilidad de establecer leyes (causales o estadísticas); las posiciones más abiertas aceptarán todas las opciones de explicación que se plantean a continuación, tomando como control de la validez otros factores (la eficacia cognitiva, la capacidad de superar contrastaciones, etc.). A continuación expondremos algunas de ellas, atendiendo a la clasificación de Hempel (1986: 233 y s.):

1. Explicación nomológico-deductiva: es la fundamental para la concepción dominante entre los científicos. En este tipo de explicación se establece una relación entre causa y efecto de tipo necesario (siempre que se dé la causa, se producirá el efecto), estas explicaciones buscan establecer modelos legales del tipo siguiente: "cuando son satisfechas tales y tales condiciones, siempre ocurre este tipo de cosas» (Hospers 1982: 288).

En todo esquema de explicación científica, de este tipo, habría que distinguir en la argumentación dos tipos de componentes: primero las condiciones antecedentes, que pueden describir las condiciones iniciales en las que se produce la formulación de un problema que hay que explicar, junto a las condiciones de observación; un segundo componente estaría integrado por aquellas leyes científicas que permiten explicar el fenómeno. La explicación estaría constituida por la deducción a partir de ambos componentes: condiciones iniciales, observación y leyes que se aplican necesariamente. Según Bunge, lo que pretende este tipo de explicación, es decir, 
«que los sucesos no ocurren caprichosamente sino según leyes, y que no surgen de la nada, sino que emergen de condiciones preexistentes (Bunge 1972: 293).

2. Explicación estadística. También estudiada por Hempel, siguiendo el mismo esquema anterior. La diferencia es que al menos una de las leyes debe ser estadística. Una explicación estadística debe mostrar que el enunciado explicado ha de tener una elevada probabilidad de ser cierto. Se podrán establecer relaciones estadísticas derivadas de experimentación que pretenderán establecer relaciones de causalidad, así como relaciones estadísticas derivadas de estudios no experimentales (encuesta y registros estadísticos), en este segundo caso se hablará de relaciones de asociación.

La explicación nomológica, así como la estadística, están basadas en la experimentación, la cual es la única que puede demostrar la causalidad. ${ }^{3}$ Pero, es evidente que si sólo se acepta dicha metodología, ${ }^{4}$ se condena a la irracionalidad a una amplia gama de argumentaciones. La alternativa es aceptar un estatus diferenciado para las ciencias sociales, basado en las características también diferentes que poseen, desarrollando sus explicaciones mediante las explicaciones nomológicas y estadísticas, pero también a partir de las siguientes formas de explicación que se verán a continuación.

3. Explicación por procesos. Se basa en la reconstrucción completa de un proceso tipificado, para lo que deben identificarse todos los estados evolutivos o fases, seleccionando aquellos que, hipotéticamente, tienen relevancia para el desarrollo del fenómeno. Se estudian desde esta perspectiva los procesos de socialización, inadaptación, etc.

4. Explicación funcional, se refiere a un tipo particular de explicación de los acontecimientos de las ciencias sociales. Se trata de explicitar las funciones que en un sistema cumple un elemento, si se comprende el sistema se pueden explicar las funciones de sus elementos. Por ejemplo, se puede explicar funcionalmente la formación de subgrupos en una clase, a partir de las funciones de apoyo, refuerzo, referencia, etc. que cumplen los mismos. Para la explicación funcional se debe describir el fenómeno en su contexto, identificar los elementos relevantes de dicho contexto y las relaciones que establecen, la estructura del sistema (cómo es) y su dinámica (cómo funciona-actúa).

5. Explicación crítico-racional. Se pretenden identificar los aspectos intencionales de la actividad humana, establecer los intereses, motivaciones, intenciones, etc. que dan cuenta de determinados fenómenos, explicando a partir de las teorías que han mostrado su eficacia cognitiva hasta el momento. La explicación comparativa sería de este tipo: en parte funcional (comparación de estructuras y sistemas) y en parte crítico-racional (comparación del

3. No entramos aquí en el debate sobre la causalidad originado por Hume y que ha seguido hasta nuestros días. Para una discusión de las posiciones dominantes puede consultarse el texto, ya clásico, de Bunge sobre el tema (1972).

4. Debe tenerse presente que la metodología quasi-experimental no ofrecería las garantías de validez y fiabilidad que requiere una posición positivista radical. 
sentido social y cultural de los fenómenos que se explican). Se trata de explicaciones que requieren información contextual (de qué sistema se trata) y de procesos deliberativos en las comunidades científicas de referencia (teorías eficaces), hasta que se consigue establecer consensos para explicar los fenómenos.

6. Explicación teleológica. Se explica por causas finales, de lo que se trata es del análisis de la adecuación de medios a fines. Se explican las acciones o sucesos actuales por las finalidades planteadas, siendo las acciones los medios que se desarrollan para lograrlas. Tiene que ver con la finalidad de las acciones actuales; si se comprende la finalidad se pueden explicar las acciones (por ejemplo, se estudia para conseguir un título). Parecen implicar la noción de intención o de deseo, aunque el propio sujeto no sea plenamente consciente de por qué hace lo que hace. Esta implicación limita su ámbito de aplicación a las ciencias sociales y, en menor medida, a las ciencias de la vida.

El término «teleológico» es un término técnico. La teoría de los juegos (Von Neumann), por ejemplo, fundamenta sus explicaciones sobre este tipo de explicación, desarrollando el concepto de estrategia, después aplicado en el conjunto de las ciencias sociales (pedagogía, psicología, sociología, economía). Se parte de la identificación de objetivos del sujeto de investigación (individuos, grupos, organizaciones, etc.), es decir, de identificar alguna cosa pretendida o la meta de la actuación. Otra premisa se refiere a algún acto o actos conducentes a su logro (estrategia), algo así como un medio dirigido a tal fin; por último, la conclusión consiste en el empleo de este medio para alcanzar el fin en cuestión.

La diferenciación entre los dos primeros tipos de explicación y los cuatro últimos plantea varios problemas, entre los que se pueden destacar tres: la posibilidad de la predicción; el desarrollo de metodologías cualitativas y la explicación de acontecimientos singulares. Una de las consecuencias de la diferenciación se observa en relación a la capacidad predictiva de los diversos tipos de explicación. Las explicaciones causales (nomológica y estadística) permiten predicciones seguras o de alta probabilidad, pero ¿qué tipo de predicciones permiten las otras explicaciones? Permiten predicciones de baja potencia, en términos de seguridad, pero de aceptación variable según el consenso de la comunidad científica. Todos los fenómenos complejos (multivariables y basados en cadenas causales no lineales), en los que hay implicados dilatados procesos, como el aprendizaje, la motivación o la maduración psicosocial, por ejemplo, implican explicaciones procesuales o de otros tipos (funcionales, etc.). En estos casos, sólo fragmentos muy simples pueden ser explicados nomológica o estadísticamente con seguridad, y se suele tratar sólo de las condiciones necesarias para que se den dichos fenómenos, pero no del conjunto de las condiciones que los explican. El mantenimiento en el ámbito de la racionalidad científica de las argumentaciones que explican dichos fenómenos, depende de la aceptación de dichas explicaciones. Sus predicciones, aun siendo sólo estimativas, suelen 
ser bastante fiables, en términos de argumentación racional y de certeza, la manera de controlarla, como dice Popper, es la contrastación de su eficacia cognitiva.

La aceptación científica de las diversas opciones de explicación permite el desarrollo de los enfoques cualitativos, antes rechazados por pseudocientíficos o especulativos. ${ }^{5}$ En los últimos tipos de explicación (por procesos, funcional, crítico-racional y teleológica), se introducen algunos aspectos de la tradición hermenéutica: el de la explicación completada por la comprensión, dando lugar a una diferenciación epistemológica apreciable y a nuevas metodologías de investigación. La negación de estas metodologías implica replantearse completamente ciencias completas como la antropología.

Otro problema es el de la explicación de los acontecimientos singulares. Popper plantea una diferencia, en las ciencias sociales, entre la explicación de acontecimientos singulares y la de fenómenos o tipos generales de acontecimientos. La diferencia consiste en que los singulares se pueden explicar sin recurrir a un modelo, mientras los otros requieren modelos explicativos, ${ }^{6}$ construidos a partir del análisis situacional o de otras maneras. La limitación de las explicaciones singulares es la predicción, la eficacia cognitiva de las explicaciones no nomológicas ni estadísticas no permite la predicción de acontecimientos singulares. Este problema ha sido replanteado en el contexto del individualismo metodológico.

A continuación, se presenta el debate entre el individualismo metodológico y la explicación funcional reformada como uno de los debates sobre las modalidades de explicación, prestando atención a las respuestas que aportan a los problemas planteados en esta sección. ${ }^{7}$

\section{El individualismo metodológico de Jon Elster y la crítica de los modelos explicativos holísticos}

Desde el individualismo metodológico se ha mantenido que la base de las ciencias sociales son los actos de los sujetos, de tal manera que ya que los únicos que actúan y tienen intenciones son los sujetos, debe evitarse la pretensión de analizar las entidades supraindividuales como agentes sociales. La causalidad procede de lo individual a lo social; se ejerce a partir de los agentes humanos que tienen, por tanto, un papel explicativo fundamental. En cualquier caso son las unidades elementales, los actos de los individuos, los que permiten defi-

5. La compatibilidad, en ciencias sociales, de las diversas formas de explicar se basa en la necesidad de dar respuestas racionales a preguntas de muy diversos tipos.

6. Los modelos se basan en la aplicación del principio de racionalidad administrado por comunidades científicas y desarrollado a partir de la contrastación, en condiciones controladas, y la potencial falsación.

7. No se incluyen otras tradiciones de debate sobre la explicación representadas por R. Nozick (1981), siguiendo a Hempel; B.J. Caldwell (1982, 1991), siguiendo a Popper; y otros autores, por motivos de espacio. 
nir el origen de los hechos sociales entendidos como resultados de la acción de dichos agentes:

Un hecho es una instantánea temporal de una corriente de acontecimientos o una serie de tales instantáneas. En las ciencias sociales los acontecimientos elementales son las acciones humanas individuales, incluidos los actos mentales como la formación de la creencia (Elster 1990a: 13).

Pues bien, esta es la posición del marxismo analítico más reciente, pero tiene precedentes claros. En el conjunto de la filosofía de la ciencia son los positivistas los que han defendido dichas posiciones, aunque tal vez, en el siglo XX, esa adscripción genérica sea parcialmente injusta, ya que otras corrientes teóricas también se pueden identificar como defensores del individualismo metodológico. Pero los fundamentos teóricos del individualismo metodológico, en el siglo XX, indiscutiblemente se relacionan con los esfuerzos del positivismo lógico por fundamentar las explicaciones científicas en unidades elementales que pudieran ser analizadas plenamente. Sin necesidad de entrar en matices que tendrían difícil cabida en un trabajo de esta naturaleza, es posible resumir la cuestión afirmando que la herencia positivista en la tradición de las ciencias sociales se afianza sobre tres principios básicos, conforme a la concepción preconizada por quienes la han consolidado:

a) Existe un componente ontológico según el cual es preciso admitir que la realidad existe de forma independiente y está regida por leyes inmutables; en consecuencia, el conocimiento debe predecir y explicar los fenómenos mediante el conocimiento de esas leyes.

b) Hemos de partir de un componente epistemológico, según el cual el investigador debe adoptar una postura de distancia, no interactiva, procurando que los valores y juicios propios no interfieran en la obtención de resultados.

c) Finalmente, el componente metodológico exige que las hipótesis sean sometidas a pruebas empíricas bajo condiciones contrastadas por medio de la experimentación empírica manipulativa. (Guba 1990)

En el positivismo dominante hasta bien entrados los años setenta del siglo XX, se daban estos tres componentes claramente entrelazados, aunque las reformas radicales de Popper produjeron un importante cambio, manteniendo una concepción en la que el componente epistemológico estaba claramente relacionado con el metodológico. En el individualismo metodológico, especialmente, en el marxismo analítico, tampoco se daba esa relación entre los tres componentes, ya que, siguiendo a Popper, pretendían que sólo se mantuviese una relación sistemática entre la dimensión epistemológica y la metodológica. Se trataría de una reflexión sobre la manera de explicar los hechos sociales y no sobre la composición ontológica del universo social, o sobre la búsqueda de una legitimación o justificación de las instituciones sociales a partir de una concep- 
ción individualista. Este presupuesto puede ser discutible, y veremos que es una de las principales críticas al individualismo metodológico, justamente por la pretensión de ser sólo metodológico (Galeotti 1988).

La más reciente defensa del individualismo metodológico surge en el contexto de la filosofía analítica anglosajona en los años setenta, en torno a una lectura del marxismo de carácter radicalmente opuesta a la althusseriana. En ella se plantea la revisión de la teoría social marxista entendiendo que hay que reconstruirla sobre nuevos presupuestos metodológicos. Se constituye así lo que se conoce como marxismo analítico.

Aunque el marxismo analítico se sitúa en la tradición del individualismo metodológico, no se reduce a ella, como señala D. Little (1991: 183). Esta revisión da paso a un programa de investigación de los fundamentos metodológicos de las ciencias sociales, que trata de comprender los principios básicos que subyacen a estas ciencias a partir de la filosofía analítica y la ciencia social positivista, con aplicaciones concretas a partir de la teoría de la elección racional y la teoría de los juegos. Se insiste en la necesidad de la búsqueda de fundamentos en general y de microfundamentos en particular para las ciencias sociales. El objetivo fundamental es sacar a las ciencias sociales de la situación a las que las han llevado las insuficiencias del estructural-funcionalismo. Dichas insuficiencias serían:

1. El bajo rendimiento explicativo y predictivo de las teorías sociales.

2. El abandono de la metodología de la investigación científica en favor de postulados metafísicos alejados de la práctica científica habitual, con el consiguiente alejamiento entre la teoría y la investigación social.

3. La proliferación de conceptos generales con vagas referencias a propiedades estructurales o funcionales de entidades supraindividuales.

Por eso es necesario llevar a cabo un análisis crítico del estatuto de las teorías, los conceptos y las explicaciones sociales, aplicando las pautas de rigor conceptual de la filosofía analítica de la ciencia para replantear las cuestiones señaladas. Esto supone que los conceptos de cualquier teoría social deban ser precisados operativamente. También es necesario que se vuelva a las leyes entendidas como generalizaciones basadas en explicaciones científicas. Finalmente, los modelos explicativos deben ser causales, en el sentido fuerte de las ciencias naturales, superando las explicaciones no deterministas o explicaciones que no hagan necesarias las conclusiones.

8. «La más controvertida de las afirmaciones que se exponen en esta obra es la negación del papel de la explicación funcional en las ciencias sociales» (Elster 1990b: 23). Para una amplia crítica de la explicación funcional en ciencias sociales se puede consultar Elster 1990b: 48-64.

9. Como puede verse el nivel de exigencia es diferente si en lugar de solicitar «hipótesis comprobables» (Elster), que requieren previamente de rigurosas verificaciones, se solicitan «hipótesis contrastables» (Popper), que se mantendrán si superan futuras contrastaciones. 
El desacuerdo con el estructural-funcionalismo se centra en la explicación funcional. El programa de los microfundamentos sostiene que la explicación funcional en ciencias sociales es inaceptable. ${ }^{8}$ Sólo podría considerarse parcialmente aceptable si se basara en hipótesis comprobables, ${ }^{9}$ tal como aporta la biología (Elster 1984). Como señala el mismo autor: "De hecho, la explicación funcional en la biología sólo está justificada cuando creemos en la verdad de una determinada teoría causal, que es la teoría de la evolución por selección natural» (1990b: 22).

Para que la explicación sea válida es necesario tener alguna idea de los mecanismos causales que establecen y preservan la relación funcional. Pero como en las ciencias sociales no existe una teoría de generalidad comparable, el verdadero mecanismo debe ser explicado en cada caso particular (Elster 1990b: 23).

Elster, reconstruyendo la estructura reformada de la explicación funcional realizada por Cohen (1984), su principal adversario teórico en el ámbito marxista, junto a Van Parijs (1984) y Woods (1987), ha intentado demostrar dicha insuficiencia teórica y metodológica. Según Elster, Cohen afirma que una institución o pauta de comportamiento $X$ es explicada por su función $Y$ para el grupo $Z$, si y sólo si $Y$ mantiene a $X$ mediante un círculo de retroalimentación que pase por $Z$. Una explicación funcional válida que sostenga dicha tesis, debe cumplir los siguientes cinco requisitos (1990b: 55):

1. $Y$ es un efecto de $X$.

2. $Y$ es beneficioso para $X$.

3. $Y$ no es intención de los actores que realizan $X$.

4. $Y[\ldots]$ no es reconocida por los actores en $Z$.

5. $Y$ mantiene a $X$ por un giro de retroalimentación causal que pasa a través de $Z$.

En cambio, en el caso de las explicaciones funcionales sociales, según Elster, no hay manera de establecer el círculo de retroalimentación en términos suficientemente detallados para hacer válida la explicación funcional. No se dispone de hipótesis que permitan formular la historia causal de los mecanismos implicados en la producción y pervivencia de $X$. Ello exigiría que, para justificar la afirmación de que $X$ (la danza de la lluvia, o la disciplina) existe por sus consecuencias beneficiosas ( $Y=$ reforzar la cohesión social, o el orden en un grupo) para una sociedad $Z$ (el sistema social Hopi, o el grupo clase), se debería demostrar que si Y no se produce (efecto no intencional), entonces no se seguirá manteniendo $X$ (pauta de conducta intencional). Si no se pudiera demostrar esa vinculación (retroalimentación causal no demostrada), entonces no se ofrecería una correcta explicación funcional.

Según Elster, no hay manera aceptable de explicar cómo un efecto no intencional (la cohesión, o el ambiente de la clase) afecta a la producción y pervivencia de una pauta de conducta colectiva (la danza de la lluvia, la disciplina), que es llevada a cabo por motivos intencionalmente precisos (provocar lluvia, mantener el orden), sin los cuales tal conducta no se habría producido. Para Elster, 
las explicaciones funcionales en biología satisfacen las cinco condiciones antes señaladas, gracias a que existe un mecanismo de retroalimentación claramente definido, pero no sucede lo mismo en ciencias sociales, por lo que las explicaciones que se obtienen son falaces, aunque hay algunos pocos casos de explicación funcional satisfactoria en ciencias sociales la mayoría de los cuales fallan por solamente postular la condición 5 en vez de demostrarla. Sostiene Elster que «los sociólogos funcionalistas afirman como si [...] el criterio 5 se cumpliera automáticamente cuando se cumplen los otros criterios» (1990b: 56). Esta pretensión, según Elster, ${ }^{10}$ no ha podido fundamentarse; ahora bien, si cambiamos el ejemplo utilizado, pasando a un ejemplo más cercano al marxismo, la perspectiva es completamente diferente. Así, la afirmación de que $X$ (organizarse políticamente por parte de diversos grupos sociales) existe por sus consecuencias beneficiosas $Y$ (garantizar la defensa de sus intereses) para cada uno de los grupos que se organizan en $Z$ (una sociedad concreta) parece perfectamente aceptable como explicación de cómo un efecto no intencional (defensa efectiva de intereses) afecta a la producción y pervivencia de una pauta de conducta grupal (organizarse), que es llevada a cabo por motivos intencionalmente precisos (conseguir más fuerza), sin los cuales tal conducta no se habría producido. Aunque puede pasar, en algunos casos, que aun organizándose políticamente no se consiga una efectiva defensa de los intereses de grupo.

En cualquier caso, en esta reformulación del ejemplo, en el caso de la organización política, se incluye uno de los aspectos que la explicación funcional no marxista obviaba, es decir, se incluye una historia causal que permite comprender la persistencia de la organización o sus variaciones en el tiempo, de manera que se muestra cómo la defensa efectiva de intereses determina esa opción de conducta social. Los efectos esperados, criticados por Elster, son consistentes gracias a la disponibilidad del análisis histórico (Little 1991: 95).

Sin entrar ahora en el debate sobre las funciones latentes (Merton [1949] 1980, cap. III: 92-160), parece que Elster mezcla los «efectos efectivos» con los «efectos esperados» que motivan la acción colectiva, en este caso la organización. En este punto hay que recordar que la explicación funcional teleológica sólo pretende incluir los efectos esperados. ${ }^{11}$ Los argumentos de Elster se fundan en ejemplos extraños, en algunas ocasiones en la tradición explicativa del marxismo, ${ }^{12}$ o distorsionadores que los convierten en ridículas argumentaciones descontextualizadas. Sin embargo, la llamada de atención sobre la importancia de los actores intencionales, así como la reflexión sobre los microfundamentos ha sido muy relevante. En definitiva, la tesis de Elster es que los fenómenos

10. Este mismo ejemplo es discutido por Cohen (1986: 289, 305...)

11. La explicación teleológica subyace en muchas de las explicaciones funcionales y es razonable criticar su uso solapado (Ryan 1970: 195-221), pero eso no significa que no se pueda explicar teleológicamente. Si no cupiera esa posibilidad, ¿̨cómo, por ejemplo, podría explicarse por qué un universitario estudia durante cinco años una carrera? Si se excluye un «efecto esperado» futuro, la explicación es parcial y cuesta explicar esa dedicación.

12. Tal como ha destacado Wood (1987). 
funcionalmente explicados han de poder ser también explicados causalmente, y que para eso es necesario prestar atención a los fundamentos de la explicación social, en especial a la explicación intencional (1990b: 65-81).

Como se verá, la explicación intencional está en estrecha relación con el individualismo metodológico. Dado que las ciencias físicas y biológicas no explican la conducta intencional, "la explicación intencional es la característica que diferencia las ciencias sociales de las naturales» (1990b: 65). Explicar intencionalmente es equivalente a demostrar que la conducta intencional es realizada para lograr una meta. En ese sentido, se explica una acción intencional cuando es posible especificar el estado futuro que se pretendía con la acción (1990b: 66). La explicación intencional incluye acción, deseo y creencia. Sin embargo, Elster reconoce que la explicación intencional no permite una completa explicación de la conducta, ya que deja sin explicar las creencias y los deseos y se concentra en las razones que orientaron la acción. ${ }^{13} \mathrm{La}$ explicación intencional debe mostrar que la acción se llevó a cabo por una razón concreta y particular y excluye todo aquello que no tiene que ver con los propósitos del agente racional. Esa reducción permite desarrollar una explicación por mecanismos mucho más cómoda, pero significa una petición de principio excesiva para ser obviada.

La propuesta de Elster pretende mostrar que algo característico de las ciencias sociales es su imposibilidad para formular leyes, su carácter no universal, sino sólo individual y local. Como plantea Little, se reducen a hablar de «hechos acerca de agentes individuales» (1991: 18). Según este planteamiento, los actores humanos, en sus acciones, muestran ciertas regularidades, pero regularidad no es lo mismo que universalidad. Esa reducción de la potencia explicativa a un conocimiento circunstancial y, como máximo, probabilístico, obliga a replantear la capacidad teórica de las ciencias sociales y, de forma más notoria, entre las tradiciones con más ambición teórica como el marxismo.

\section{La explicación por mecanismos}

Para los individualistas metodológicos y para otras reformulaciones contemporáneas de las ciencias sociales, especialmente para los marxistas analíticos, el marxismo, si quiere conservar su lugar como paradigma teórico de las ciencias sociales, no puede seguir siendo una «teoría de la historia sin teoría acerca de las acciones de las personas que hacen esa historia» (Przeworski 1987: 104). La consideración de las acciones de los individuos como puntos clave de la explicación de los hechos sociales se convierte en un desafío para la teoría marxista de la historia y, en general, para toda teoría que excluya, de manera sistemática, en la construcción de sus explicaciones, la intervención de la intencionalidad así como de otros elementos subjetivos.

13. Elster ha intentado superar esas limitaciones en obras posteriores (1995 y 1997), pero la crítica de su teoría de los mecanismos que subyacen a la formación de deseos y creencias requiere un trabajo monográfico. 
La propuesta del marxismo analítico se concreta en la necesidad de investigar las condiciones del establecimiento de explicaciones causales en las ciencias sociales. Para ello es necesario investigar los microfundamentos de la explicación social, detectando los mecanismos causales implicados en la ocurrencia de los fenómenos a partir de los cuales sería posible la formulación de explicaciones causales. Tales explicaciones no pueden desarrollarse con el modelo nomológico deductivo, por el simple hecho de que no se dispone de leyes sociales que cumplan los requisitos exigidos por el modelo, pero sí pueden establecerse en términos menos rigurosos. La búsqueda de los microfundamentos para las explicaciones en ciencias sociales, búsqueda basada en el supuesto de que los fenómenos sociales son plenamente comprensibles como resultado de las acciones de los individuos, es lo que caracteriza al individualismo metodológico.

Las ciencias sociales, según Elster, deben poner el acento en los mecanismos antes que en las leyes, siendo conscientes de las limitaciones que esta opción impone:

Las ciencias sociales [...] Lo que menos frecuentemente son capaces de hacer es expresar las condiciones necesarias y suficientes en las cuales se ponen en funcionamiento los diversos mecanismos. Hay otro motivo para acentuar los mecanismos antes que las leyes. Por su naturaleza las leyes son generales y no sufren excepciones. No se puede tener una ley en el sentido de que «si $p$, entonces q». En contraste, los mecanismos no tienen ninguna pretensión de generalidad (1990a: 19).

En lugar de leyes se deben intentar identificar mecanismos del tipo «si $p$, entonces q", de tal manera que se pueda hacer progresar el conocimiento con formulaciones limitadas de ese estilo. En ciencias sociales es necesario identificar los factores causales implicados en la ocurrencia de los fenómenos. Con ello se permitiría la elaboración de explicaciones adecuadas, de situaciones y hechos que por ahora sólo están limitados a imprecisas explicaciones funcionales. Se trata, según Elster, de retomar la idea de explicar un acontecimiento atendiendo a las causas, y esto implica algún relato del mecanismo causal que relaciona los dos acontecimientos. Por tanto, si se puede dar cuenta de los mecanismos implicados, se habrá avanzado en la dirección correcta en el terreno de la explicación social: «explicar un acontecimiento es dar un relato de por qué sucedió. [...] citar un acontecimiento anterior como la causa del acontecimiento que se desea explicar, junto con algún relato del mecanismo causal que relaciona los dos acontecimientos» (1990a: 13).

La investigación en torno a la identificación de los mecanismos causales supone, entre otras cosas, un procedimiento analítico dirigido a la indagación en los niveles elementales de la realidad social en los que se configura la microfísica causal de los fenómenos sociales. Se trata de explorar los fundamentos de los fenómenos sociales para poder establecer los mecanismos a partir de los cuales tales fenómenos son explicables, sea cual sea su grado de complejidad. 
Elster (1990a: 15-18) es claro al respecto, pues afirma que es preferible explicar lo macro por lo micro que lo macro por lo macro; es más eficaz abrir la caja negra y ver el funcionamiento de los mecanismos de los fenómenos, ya que así se reduce el riesgo de confundir explicación y correlación, pudiendo, al mismo tiempo, detectar los antecedentes causales de los fenómenos.

Es necesario dar una explicación de los mecanismos a través de los cuales se configuran los fenómenos que se pretenden explicar. La explicación no puede reducirse, por ejemplo, a la afirmación de G.A. Cohen de que la tendencia de las fuerzas productivas a desarrollarse se explica porque los individuos, siendo inteligentes y racionales, y encontrándose en una situación de escasez material, tienen el interés en, y la capacidad de, realizar los cambios infraestructurales y estructurales necesarios para que lo disfuncional sea sustituido por lo funcional y el progreso socioeconómico e histórico continúe. La explicación, según Elster, si queremos predecir los cambios posibles y la dirección de los mismos, exige dar cuenta de los distintos problemas de acción colectiva que puedan presentarse, así como su forma de operar, que por cierto no tiene por qué ser la propuesta por G.A. Cohen, ya que el progreso puede no continuar.

En una explicación, cuando el explanandum es un hecho social, ha de ser posible indicar las causas a través de las que se produce ese hecho. Como señala Elster, sin un profundo conocimiento de los mecanismos que actúan en el micronivel, las hipótesis marxistas sobre las macroestructuras y el cambio a largo plazo están condenadas a permanecer en un mero nivel especulativo.

Para los individualistas metodológicos, los fenómenos sociales complejos son agregados que pueden descomponerse en partes más simples. Por eso hay que ir a los microfundamentos, a las unidades básicas de lo social, a su articulación y su relación, intentando identificar los mecanismos a través de los que operan. De esta manera, se podrán establecer explicaciones eficaces aunque sean de bajo nivel. Esta limitación no excluye la posibilidad de desarrollar en algún momento teorías de carácter más general. En la medida en que se consigan buenos análisis de los mecanismos se encontrarán regularidades al más bajo nivel, explicables desde teorías más fundamentales a través del establecimiento de las leyes que los rigen. En este caso, el análisis de los microfundamentos es esencial si se quiere llegar a una descripción de los fenómenos sociales en tanto que gobernados por leyes. Por tanto, la tesis de los microfundamentos conduce a preguntarnos por las unidades básicas a través de las que opera la causalidad, y en las que se basa la explicación. Tales unidades deben ser identificables en términos de acción individual y de interacción desde donde se surgen y se configuran los fenómenos sociales más complejos.

La acción es, pues, entendida como unidad básica de lo social en cuanto acción intencional, es decir, en cuanto acción dirigida a un fin, o hacia algún objetivo que el sujeto quiere alcanzar. Desde luego, hay acciones no intencionadas subproducto de otras que interesan para explicar ciertos hechos, pero aquí nos ocupamos sólo de las primeras. Dicha hipótesis significa que las acciones son significativas para el agente. No sólo se procesa información manipu- 
lando signos internos, sino que además tenemos, a diferencia de los ordenadores, acceso al significado de estos símbolos.

La acción no es evaluada como racional en sí misma, sino como medio más o menos eficaz para un fin. Este planteamiento es similar a la racionalidad instrumental de Habermas o a la racionalidad de medios de Weber, pero discrepa profundamente de la racionalidad sustantiva de fines. Hay, por lo tanto, en la explicación por mecanismos, una cierta concepción de la racionalidad que es consecuencia de la consideración de la acción en el margen estrecho de las condiciones empíricas de la acción de los sujetos humanos concretos. Los modelos de racionalidad normativa, o de ética normativa, no tienen cabida cuando se habla de sujetos concretos limitados en su capacidad para actuar y analizados según un modelo de acción racional orientada a los resultados (Elster 1991: 120-121). Si se explica el mecanismo mediante el cual actúan las normas de la siguiente manera: las normas determinan las preferencias; las preferencias más el conjunto factible determinan las acciones (1978; 1991: 119-129), entonces se reduce su análisis a sus "efectos efectivos» y se consideran las «metas y creencias basándonos en acciones y suponiendo además que la gente es por lo común racional» (Elster 1991: 120). Este planteamiento considera la acción desde una perspectiva meramente instrumental, no hay una racionalidad normativa sino un cálculo de opciones, beneficios e inconvenientes.

La consecuencia metodológica es que se deben superar los modelos de Hempel o Popper, pero también los marxistas, en los que la racionalidad normativa se utiliza como principio explicativo, ${ }^{14}$ lo que significa admitir que los sujetos no son racionales en un sentido que supere el mero cálculo, sino que están sujetos a mecanismos afectivos y cognitivos que suponen distorsiones de diversos tipos que les hacen con frecuencia imprevisibles o, como mínimo, inadecuados para cumplir las condiciones que exigen las teorías de la racionalidad de los modelos holistas.

\section{La explicación funcional reformada en el materialismo histórico}

Desde los planteamientos antirreduccionistas ${ }^{15}$ se ha considerado que no hay posibilidades de explicación a partir de los elementos básicos. El presupuesto de que el todo es más que la suma de las partes es una de las cuestiones que

14. Como se puede ver, en este tema concreto, hay una importante discrepancia entre Hempel y Popper, respecto de los individualistas metodológicos posteriores. Hay que considerar que Popper aceptaba que las creencias de los sujetos pueden ser incompletas, erróneas o falsas, produciendo resultados no esperados por el sujeto, pero que eso no significaba ninguna merma en su racionalidad, ya que en cualquier caso habría realizado una elección basándose en una evaluación de su información, eligiendo la alternativa aparentemente óptima (Popper 1997: 153-179).

15. Aceptando la diferenciación de opciones explicativas propuesta por Levine et al. (1987), a partir de ahora se considerará el marxismo funcionalista de Cohen, Woods y otros como antirreduccionismo más que como holismo. 
sustentan las tesis de que son las entidades supraindividuales las que permiten explicaciones en las ciencias sociales. Las acciones individuales y las características de los actores y colectivos se explican en función de las propiedades de tales entidades. Lo individual es contingente, por lo que la explicación debe formularse en términos de procesos, estructuras y funciones. Pues bien, en este contexto G. Cohen desarrolla sus trabajos sobre el materialismo histórico en el marco del marxismo anglosajón, superando u obviando buena parte de las preocupaciones derivadas del hegelianismo marxista. Su defensa de la racionalidad del materialismo histórico se desarrolla desde la más escrupulosa terminología, respetando los principios de claridad conceptual y terminológica característicos de la filosofía anglosajona. Dicha defensa se desarrolla como una reflexión sobre la sociedad y la historia, superando los meros debates metodológicos característicos del estructuralismo althusserista, aunque reconociendo la importancia de sus aportaciones en la recuperación del interés por el materialismo histórico.

La más importante aportación de Cohen es el reconocimiento del modelo explicativo marxista como un modelo de explicación funcional. La explicación, por ejemplo, de la superestructura social por la estructura económica, y de ésta por el grado de desarrollo de las fuerzas productivas, son explicaciones de dicho tipo. Es una explicación funcional porque explica la existencia en una determinada formación social de unas determinadas relaciones de producción por el efecto (positivo) que dichas relaciones de producción son capaces de generar para el desarrollo de las fuerzas productivas.

La propuesta de Cohen puede ser considerada como un modelo de explicación reformado porque supera claramente las concepciones de Malinowski, pero también las más elaboradas por Merton. El modelo clásico de Merton consideraba que no se podía mantener el análisis holístico clásico, es decir, la consideración de la sociedad como un todo unificado. Por su parte, Elster define la explicación funcional en su artículo de 1987:

La explicación funcional es una subvariedad de la clase general de las explicaciones de consecuencia. Éstas explican los fenómenos sociales en función de sus consecuencias reales, en oposición a la explicación por las causas (explicación causal) o por las consecuencias intencionadas (explicación intencional). El rasgo distintivo de las explicaciones funcionales es que las consecuencias explicativas son beneficiosas para alguien o para algo (Elster 1987: 26).

La primera crítica posible contra una explicación funcional de este tipo es que parece dar cuenta de un hecho por sus efectos, es decir, explicar la aparición de un fenómeno por las posteriores consecuencias de dicha aparición. Esta crítica sólo puede darse a las aplicaciones más ingenuas de la explicación funcional. Sin embargo, en la obra de Cohen, hay una respuesta clara. En primer lugar, una explicación funcional nunca explica la aparición de un fenómeno, sino su persistencia. Una explicación de la existencia de las religiones en las sociedades primitivas, o una cierta aplicación curricular, en un caso 
educativo, no pretende dar cuenta de cómo se formaron tales religiones, o la génesis del formato curricular, sino de la razón por la que están universalmente presentes: mantienen la cohesión social, o permiten en su caso el éxito escolar.

En segundo lugar, una explicación funcional no da cuenta de la presencia de un fenómeno por sus efectos, sino por su capacidad para producir tales efectos. La premisa mayor de un razonamiento funcional es un condicional disposicional: si para un determinado nivel de desarrollo de las fuerzas productivas existe un conjunto de relaciones de producción óptimo para la potenciación de su desarrollo posterior, estas relaciones de producción serán las realmente existentes en la formación social en cuestión porque son las más funcionales (Cohen 1986: 275-306).

Otra crítica contra las explicaciones funcionales remite a la dificultad de su contrastación empírica. Nagel y Hempel no discuten la validez general de las explicaciones funcionales; por el contrario, es posible reconstruir una explicación funcional en términos nomológico-deductivos siguiendo este esquema (Cohen 1986: 300 y s.):

a. En un momento dado $t$, un sistema $s$ funciona adecuadamente en unas condiciones de entorno $c$.

b. Un sistema de tipo $s$ sólo funciona adecuadamente en unas condiciones $c$ si se cumple una condición necesaria $n$.

c. Si el rasgo $r$ se diera en $s$, entonces, como consecuencia, se cumpliría la condición $n$.

d. Luego en el momento $t$ el rasgo $r$ está presente en $s$.

Pero, está claro que la inferencia anterior sólo es válida si existe un único $r$ que satisface $n$. Si por el contrario, contamos con un conjunto $R$ de rasgos (alternativas funcionales) que satisfacen $n$, sólo podemos afirmar que alguno de estos rasgos estará presente en $s$ en el momento $t$. Pero esto supone una inmediata dificultad de contrastación: ¿`ómo podemos saber que el rasgo $r$ es único? Y, si no lo es, la explicación funcional sólo consigue resultados triviales (Nagel 1979).

Una explicación funcional que ofrezca graves dificultades para su contrastación empírica difícilmente podrá servir de base para la predicción o para la formulación de generalizaciones fuertes. Pero aunque el análisis funcional pierda contrastabilidad puede seguir siendo útil como modelo heurístico. Para el investigador social no puede ser trivial comprobar que un rasgo $r$, de un conjunto dado $R$, debe estar presente, en un sistema social, para satisfacer una cierta condición necesaria para la reproducción de dicho sistema. En el peor de los casos este conocimiento permite orientar la investigación hacia la identificación de ese rasgo dentro de un conjunto de posibles alternativas funcionales y, después, buscar las razones particulares de la selección de ese rasgo concreto a partir del repertorio disponible. La hipótesis funcional, en este caso, sería, al menos, una excelente herramienta heurística para la comprensión de la sociedad objeto de estudio. 
Otra crítica, muy relevante en este trabajo, se refiere a la necesidad o no de especificar el mecanismo implícito en una explicación funcional. ¿Es legítima una explicación funcional que no esté en condiciones de especificar su mecanismo subyacente? Elster, criticando precisamente a Cohen, ha señalado que sin especificar el mecanismo causal implícito en una pretendida explicación funcional no se pueden aportar correlaciones explicativas y correlaciones no explicativas. También Maravall entiende que «es imposible distinguir entre correlaciones verdaderas y falsas sin algunas hipótesis a priori sobre el mecanismo causal en funcionamiento» (1978: 157).

La posición de Cohen es que una explicación funcional es legítima en la medida en que pueda relacionarse con un enunciado legal que concede carácter general a dicha explicación. Tal como resume Elster, la posición de Cohen es la siguiente:

Una explicación funcional debe estar respaldada por algo más allá de la mera observación de que el explanandum tiene consecuencias beneficiosas, pero no necesitamos apelar a un mecanismo específico para el respaldo. Las leyes de consecuencia proporcionan un respaldo alternativo, por ejemplo, enunciados generales legaliformes a los efectos de que cada vez que una institución o una conducta tuviera efectos beneficiosos, se observaría realmente (1990b: 61).

En cualquier caso, el propio Cohen estudia varias posibilidades de mecanismos causales dentro de la explicación funcional, a los que denomina intencional, darwiniano y lamarkiano.

a) Mecanismo intencional. El rasgo funcional sería introducido de forma deliberada y calculada por un conjunto de individuos conscientes de sus repercusiones funcionales.

b) Mecanismo darwiniano. Las instituciones sociales, surgidas por causas ajenas a la explicación funcional, son filtradas según su mayor o menor adecuación a una realidad preexistente y más sólida, como la estructura económica, siguiendo un principio análogo a la selección natural darwiniana.

c) Mecanismo lamarkiano. La institución social se adapta a los cambios en la estructura social.

Según Cohen no se puede pretender formular un mecanismo causal único y general para la explicación funcional en las ciencias sociales. Es en este sentido que se plantea la crítica de Elster: la explicación funcional sería consistente si se pudiera asegurar la existencia de cierto mecanismo de realimentación, aunque en algún caso no se pudiera demostrar, tal como hace la biología, con los presupuestos de la teoría de la evolución. Sin embargo «no hay un análogo de las ciencias sociales a la teoría de la evolución y, por lo tanto, los científicos sociales se ven obligados a mostrar en cada caso cómo funciona la realimentación» (1990b: 58). 


\section{Crítica al individualismo metodológico}

Entre las diversas críticas del individualismo metodológico se puede destacar la crítica al funcionalismo marxista reconsiderado.

La defensa de Allen W. Wood de la explicación funcional en el marxismo se basa entre otras cosas en que Elster se «apoya en una caricatura de esta clase de explicación, del mismo modo que su crítica del uso de la teleología en la filosofía de la historia se apoya en una caricatura del tipo de afirmaciones teleológicas que hizo Marx» (Wood 1987: 1-2). Además de la crítica del uso parcialmente injusto e interesado que hace Elster de Marx, Wood también considera que en algún caso Elster se equivoca, especialmente cuando describe lo que es la explicación funcional.

[...] es un error de Elster describir la explicación funcional como una explicación de la conducta que menciona simplemente el resultado de la conducta que ha de ser explicada (es decir, el beneficio que produce a algo o alguien) (Wood 1987: 7).

Para Woods se explica una conducta $C$ mencionando su contribución al resultado $R$, sólo si se ofrece una explicación funcional de $C$, lo que implica considerar que hay una disposición a que se produzca $R$, de tal manera que $C$ ocurre a causa de dicha disposición o tendencia. Las conductas alimentarias son funcionales porque permiten que el sujeto se mantenga vivo, pero mantenerse vivo es algo hacia lo cual el sujeto se ve impelido. Como dice Wood, a menos que haya realmente tal tendencia, no se produce una explicación funcional satisfactoria de $C$, sea lo que fuese lo que el individuo haya intentado hacer.

Tras estas consideraciones, Woods llega a la conclusión de que aunque parte del marxismo haya presentado sus análisis y propuestas de forma «confusa e inadecuada», eso no tiene por qué significar "que hay algún defecto en la explicación funcional como tal o en el proyecto teórico del materialismo histórico en cuanto está a favor de tales explicaciones» (Wood 1987: 11). Que Marx no presentara formalmente su metodología, que no hiciera un uso absolutamente coherente en todos sus textos - hasta en los no publicados por el propio Marx y considerados por él sólo como borradores - no significa que la única interpretación metodológicamente correcta del marxismo sea el individualismo metodológico, planteamiento claramente rechazado por Marx en sus críticas a los economistas ingleses y escoceses. Por tanto, y según Wood, el modelo explicativo dominante en el marxismo debe ser el funcionalista, básicamente porque fue el que Marx utilizó conscientemente, aunque no lo nombrara.

Otras críticas a las explicaciones exclusivamente basadas en la identificación de los mecanismos consisten en aceptar los microfundamentos desde el antireduccionismo. Entre los autores que defienden dicha posición se puede destacar a Levine, Sober y Olin Wright. Los autores citados consideran que, sea cual sea el debate metodológico, lo peculiar de la teoría de Marx son sus 
aportaciones esenciales a la ciencia social, aunque consideran que su metodología «probablemente no sea más que una buena metodología científica. Pero el individualismo metodológico no es una buena metodología científica, aun cuando [...] algunas de las intuiciones que lo motivan sean razonables» (Levine et al. 1987: 133).

Este autor, partiendo de una diferenciación de las diversas modalidades de la explicación en ciencias sociales, diferencia cuatro principales:

a) el atomismo, considerado como «una actitud metodológica que niega que las relaciones - ya sea entre individuos o entre entidades sociales - sean jamás auténticamente explicativas» (Levine et al. 1987: 135);

b) el individualismo metodológico, presentado como compartiendo con el atomismo «la idea de que las explicaciones sociales son en última instancia reductibles a explicaciones a nivel individual» (1987: 137), pero incluyendo propiedades relacionales en los mismos individuos, de tal manera que «si una propiedad social es explicativa, lo es porque es reductible a las propiedades relacionales de los individuos concretos» (1987: 139);

c) el holismo radical, para el cual las relaciones concretas entre los individuos son esencialmente epifenoménicas con respecto a las explicaciones sociales. Son generadas por la actuación del todo y, por sí solas, no explican nada (1987: 140); y

d) el antireduccionismo, el cual consideran que es su propia posición teórica, caracterizada por reconocer «la importancia de las exposiciones de micronivel para explicar los fenómenos sociales, al tiempo que admite la irreductibilidad de las exposiciones de macronivel a estas explicaciones de micronivel» (1987: 143).

Después de clarificar que no puede considerarse al individualismo metodológico como si fuera atomismo, ni tampoco al antirreduccionismo como si fuera holismo radical, las posiciones básicas quedan justamente presentadas. El debate "contra» el individualismo parte del rechazo de su pretensión de que «es deseable no añadir simplemente una exposición de microcausas a las macroexplicaciones, sino reemplazar las macroexplicaciones por microexplicaciones».

Este planteamiento es rechazado por el error básico del reduccionismo de lo macro a lo micro, el error de confundir entre muestras y tipos. Las muestras son ejemplos o casos concretos, mientras que los tipos son «las características que las muestras pueden tener en común» (1987: 144). Para Levine, Sober y Olin Wright, la mayoría de los marxistas no rechazaría la necesidad del análisis y explicación de muestras, pero, a la vez, consideraría fundamental explicar los tipos:

La distinción tipo/muestra nos permite comprender que toda ciencia tiene al menos dos tipos de proyectos explicativos: trata de explicar por qué ocurren los casos muestra y también trata de explicar la naturaleza de los tipos que entran en su campo. Por consiguiente, nos gustaría explicar por qué surgieron 
ejemplos específicos de capitalismo, cuándo y dónde lo hicieron, pero también nos gustaría explicar qué es el capitalismo (1987: 146).

Justamente eso último parece que no puede ser aceptado por el individualismo metodológico. Su proyecto reduccionista fracasa porque la ciencia no puede renunciar a la explicación que va más lejos de los casos muestra. Pero, al margen del interés por explicar en un nivel macro, debe considerarse que hay propiedades emergentes que sólo son explicables en un nivel macro, por tanto no son reducibles al micronivel.

Para Levine, Sober y Olin Wright, su crítica de las pretensiones absolutistas del individualismo metodológico no implica que los antireduccionistas no admitan la importancia de los análisis de micronivel, recordando que el antirreduccionismo no es holismo radical: «De hecho, lejos de rechazar los microniveles de análisis, la forma de antirreduccionismo que hemos descrito concede gran importancia a los "microfundamentos" de las macroexplicaciones" (1987: 149).

Con el análisis de los microfundamentos se consigue mejorar la «confianza» de las teorías, pero también se consigue mejorar su capacidad explicativa profundizando y desarrollando nuevos matices y condiciones de aplicación, además «ayuda [...] a dar un orden teórico a las categorías utilizadas en las macroexplicaciones de la teoría social. Éste, a su vez, puede facilitar la tarea de resolver las anomalías empíricas en la investigación» (1987: 154).

\section{Bibliografía}

Apostel, L. (1973). «Remarques sur la notion d'explication». En APOstel, L. (ed.). L'explication dans les sciences. París: Flammarion.

Boudon, R. (1990). L'art de se persuader des idées douteuses, fragiles ou fausses. París: Fayard.

Bunge, M. (1972). Causalidad. El principio de causalidad en la ciencia moderna. Buenos Aires: EUDEBA.

- (1973). La ciencia, su método y su filosofía. Buenos Aires: Ediciones Siglo XXI.

- (1983). La investigación cientifica. Barcelona: Ariel.

Cellerier, G. (1973). «L'explication en biologie». En Apostel, L. (ed.). L'explication dans les sciences. París: Flammarion, p. 120-131.

CoHEN, G.A. (1984). «Réplica a Elster sobre marxismo, funcionalismo y teoría de juegos». Zona Abierta 33: 22-42.

- (1986). La teoría de la historia de Karl Marx: una defensa. Madrid. Siglo XXI.

ELSTER, J. (1984). «Marxismo, funcionalismo y teoría de juegos. Alegato en favor del individualismo metodológico». Zona Abierta 33: 21-62.

- (1987). «Nuevas reflexiones sobre marxismo, funcionalismo y teoría de juegos». Zona Abierta 43-44: 25-52.

- (1990a). «Mecanismos». Tuercas y tornillos. Barcelona: Gedisa, p. 13-20.

- (1990b). «Modalidades de la explicación científica». El cambio tecnológico. Barcelona: Gedisa, p. 17-81.

- (1991). El cemento de la sociedad. Las paradojas del orden social. Barcelona: Gedisa.

- (1995). Psicologia política. Barcelona: Gedisa. 
— (1997). «Una defensa de los mecanismos». Egonomics. Barcelona: Gedisa, p. 179214.

GALEOTTI, A.E. (1988). Individualle e colletivo. L'individualismo politico metodologico nella teoria politica. Milán: Franco Angeli.

GarCÍA, R. (1973). «L'explication en physique». En Apostel, L. (ed.). L'explication dans les sciences. París: Flammarion, p. 103-119.

GubA, E. (ed.) (1990). The paradigm dialog. Newbury Park: Sage.

HeMPEL, C.G. (1986). La explicación cientifica. Estudios sobre la filosofía de la ciencia. Barcelona: Paidós.

- (1973). Filosofía de la ciencia natural. Madrid: Alianza.

HOSPERS (1982). Introducción al análisis filosófico. Madrid: Alianza.

JACOB, F. (1981). Le jeu des possibles. Essai sur le diversité du vivant. París: Fayard.

LeVINE, A.; SObER, E.; Olin WRight, E. (1987). «Marxismo e individualismo metodológico". Zona Abierta 41-42: 131-157.

LitTle, D. (1991). Varieties of Social Explanation. Oxford: Westview Press / San Francisco: Westview Press.

MARAVALL, J.M. (1978). «Sociología y explicación funcional». En JiMÉNEZ BlANCO, J.; MOYA, C. (comps.). Teoría sociológica contemporánea. Madrid: Tecnos.

MERTON, R.K. (1980). Teoría y estructura sociales. México: FCE.

Nagel, E. (1981). La estructura de la ciencia. Barcelona: Paidos.

PARIJS, Ph. van (1984). «El marxismo funcionalista rehabilitado». Zona Abierta 33: $21-42$.

Piaget, J. (1954). Problèmes de Psychologie génétique. París: Denoël-Gonthier.

- (1979). L'Épistemologie génétique. París: Gallimard.

PinILLOS, J.L. (1983). Las funciones de la conciencia. Madrid: Real Academia de Ciencias Morales y Políticas.

POPPER, K.R. (1982). La lógica de la investigación cientifica. Madrid: Tecnos.

- (1997). «Modelos, instrumentos y verdad. El estatus del principio de racionalidad en las ciencias sociales». El mito del marco común. En defensa de la ciencia y la racionalidad. Barcelona: Paidós, p. 153-179.

PRZEWORSKI, A. (1987). «Marxismo y elección racional». Zona Abierta 45: 97-136. RAPPAPORT, S. (1995). «Economic Models and Historical Explanation». Philosophy of the Social Sciences 25 (4): 421-441.

RoEMER, J. (1987). «El marxismo de la "elección racional": algunas cuestiones de método y contenido». Zona Abierta 45: 137-151.

RYAn, A. (1970). Metodología de las ciencias sociales. Madrid: Euroamérica.

- (ed.) (1976). La filosofía de la explicación social. Madrid: FCE.

Russell, B. (1981). «La filosofía del atomismo lógico». En Muguerza, J. (1981). La concepción analítica de la filosofía. Madrid: Alianza, p. 139-251.

SKINNER, B.F. (1970). Ciencia y conducta humana. Barcelona: Fontanella.

WoOD, A.W. (1987). «Materialismo histórico y explicación funcional». Zona Abierta 43-44: 1-23.

WRIGHT, G.H. von (1979). Explicación y comprensión. Madrid: Alianza. 